\title{
Adaptive Jamming Suppression in Coherent FFH System Using Weighted Equal Gain Combining Receiver over Fading Channels with Imperfect CSI
}

\author{
Yishan He, Yufan Cheng, Gang Wu, Binhong Dong, and Shaoqian Li \\ National Key Laboratory of Science and Technology on Communications, University of Electronic Science and Technology of China, \\ Chengdu 611731, China
}

Correspondence should be addressed to Yishan He; heyishan@live.com

Received 2 December 2014; Revised 4 February 2015; Accepted 9 March 2015

Academic Editor: Tho Le-Ngoc

Copyright (C) 2015 Yishan He et al. This is an open access article distributed under the Creative Commons Attribution License, which permits unrestricted use, distribution, and reproduction in any medium, provided the original work is properly cited.

\begin{abstract}
Fast frequency hopping (FFH) is commonly used as an antijamming communication method. In this paper, we propose efficient adaptive jamming suppression schemes for binary phase shift keying (BPSK) based coherent FFH system, namely, weighted equal gain combining (W-EGC) with the optimum and suboptimum weighting coefficient. We analyze the bit error ratio (BER) of EGC and W-EGC receivers with partial band noise jamming (PBNJ), frequency selective Rayleigh fading, and channel estimation errors. Particularly, closed-form BER expressions are presented with diversity order two. Our analysis is verified by simulations. It is shown that W-EGC receivers significantly outperform EGC. As compared to the maximum likelihood (ML) receiver in conventional noncoherent frequency shift keying (FSK) based FFH, coherent FFH/BPSK W-EGC receivers also show significant advantages in terms of BER. Moreover, W-EGC receivers greatly reduce the hostile jammers' jamming efficiency.
\end{abstract}

\section{Introduction}

As a powerful antijamming method, fast frequency hopping (FFH) is widely used in military applications. FFH employs a number of advantages including capability of antijamming, robustness against multipath fading, and low probability of interception $[1,2]$.

In the presence of jamming, various diversity combining schemes have been proposed for noncoherent frequency shift keying (FSK) based FFH, including maximum likelihood (ML) combining [1-7], FFT based combining schemes [8], linear combining (LC) [9], self-normalization combining [10], noise-normalization combining [11], product combining [12-17], and clipped combining [18, 19]. Among the noncoherent FFH/FSK combining schemes, ML combining yields the best BER performance in the presence of jamming.

In spite of the low complexity in implementation, noncoherent FFH systems have inevitable shortcomings, for example, performance loss due to noncoherent diversity combining. With the growing demand of better performance in antijamming communications, coherent phase shift keying
(PSK) based FFH system draws much attention. As indicated in [20] and the references therein, coherent reception has been made feasible by maintaining a continuous phase at the transmitter from hop to hop. Kang and Teh [20] studied the bit error ratio (BER) of coherent FFH/BPSK with partial band noise jamming (PBNJ) and AWGN channel. The authors considered coherent ML combining, LC combining, and harddecision majority-vote combining, which significantly outperform various noncoherent FFH/FSK diversity combining schemes in terms of BER. However, the fading channels were not considered in [20]. In the presence of fading channels, we have proposed a novel FFH scheme [21], which enables reliable channel estimation for FFH signals. And we extended the study of [20] to the Rayleigh fading channels with imperfect channel state information (CSI) [22], where we analyzed the BER of FFH/BPSK with maximum ratio combining (MRC) and equal gain combining (EGC). It is illustrated that the two combining schemes have a close BER performance in the presence of PBNJ. However, the jamming suppression was not addressed in $[21,22]$. 
This paper addresses the jamming suppression problem with coherent FFH/BPSK. In analysis, we consider PBNJ and frequency selective Rayleigh fading channels with imperfect CSI. Based on the studies of the EGC receiver [22], we give a further simplification on the BER expression. Then we propose adaptive jamming suppression schemes, namely, weighted EGC (W-EGC) with the optimum and suboptimum weighting coefficient, where the analytical BER expressions are also derived. Particularly, with diversity order $L=2$, we work out closed-form BER expressions for the EGC and WEGC. The theoretical results are validated by simulations. It is shown that the $\mathrm{W}$-EGC receivers significantly outperform the noncoherent FFH/FSK ML receiver in terms of BER. It is also shown that with the increase of signal to jamming ratio (SJR), as compared with EGC, the optimum W-EGC lowers the error floor which is determined by the signal to noise ratio (SNR). Besides, W-EGC receivers reduce the hostile jammer's efficiency, by forcing the jammer to take full-band jamming to achieve the worst case jamming.

\section{System Model}

To guarantee reliable channel estimation, the so-called subset-based coherent FFH scheme [21] is adopted, where we partition the original hopping frequency set into a number of smaller subsets and choose only one of the frequency subsets as the hopping frequency set within a frame. The frame length $T_{\mathrm{f}}$ is designed to be shorter than the channel coherence time $T_{c}$. By controlling the subset size, the hopped frequencies are revisited within $T_{c}$, which makes channel estimation feasible.

In this paper, perfect synchronization and multipath fading channels are assumed. With a hopping rate sufficiently fast, the current hop received from the second path usually falls into a posterior hop. After dehopping and filtering, only the signal from the first path will be received. Note that each modulated symbol is $L$-fold hopped and the $l$ th equivalent baseband-form received signal is given by

$$
\begin{aligned}
& y_{l}=g_{l} s+n_{l} \quad \text { without PBNJ } \\
& y_{l}^{\prime}=g_{l} s+n_{l}+J_{l} \quad \text { with PBNJ, }
\end{aligned}
$$

where $y^{\prime}$ denotes the received signal which is contaminated by PBNJ. The Rayleigh fading channel coefficient $g_{l}$ is a zero mean complex Gaussian random variable $(\mathrm{RV})$ with variance $2 \sigma_{g}^{2}$. For the $L$ hops of a modulated symbol, $g_{l}$ s are independent and identically distributed (i.i.d.). The BPSK modulated symbol is denoted by $s, s= \pm \sqrt{P_{\mathrm{d}}}$ with equal probability, where $P_{\mathrm{d}}$ is the instant power of $s$. The AWGN signal $n_{l}$ is a zero mean complex Gaussian RV with variance $2 \sigma_{n}^{2}$. The PBNJ signal $J_{l}$ is also a zero mean complex Gaussian RV, with variance $2 \sigma_{J}^{2}$. The jamming factor $\rho_{\mathrm{PBNJ}}$ is defined as the ratio of the jamming bandwidth to the entire hopping bandwidth, which is also the probability of a hop contaminated by PBNJ. Within a frame, if a hopped frequency $f_{i}$ is disturbed by PBNJ, we assume that any hop with frequency $f_{i}$ will be jammed.
Similar to [21, 22], the channel estimate is assumed to be disturbed by Gaussian errors, as

$$
\begin{gathered}
\hat{g}_{l}=g_{l}+\varepsilon_{l} \quad \text { without PBNJ } \\
\hat{g}_{l}^{\prime}=g_{l}+\varepsilon_{l}^{\prime} \quad \text { with PBNJ, }
\end{gathered}
$$

where the estimation errors $\varepsilon_{l}$ and $\varepsilon_{l}^{\prime}$ are zero mean complex Gaussian RVs with variances $2 \sigma_{\varepsilon}^{2}$ and $2 \sigma_{\varepsilon^{\prime}}^{2}$, respectively, which both are independent of $g_{l}$. We have the following decomposition between $\widehat{g}_{l}$ and $g_{l}[23]$ :

$$
g_{l}=\left(u_{l}+j v_{l}\right) \frac{\widehat{g}_{l}}{\left|\widehat{g}_{l}\right|}+\frac{R_{\mathrm{c}}+j R_{\mathrm{q}}}{\sigma_{\widehat{g}}^{2}} \widehat{g}_{l},
$$

where $R_{\mathrm{c}}$ and $R_{\mathrm{q}}$ are the second order moment between the real and imaginary part of $\hat{g}_{l}$ and $g_{l}$, as

$$
\begin{aligned}
& R_{\mathrm{c}}=\mathbb{E}\left\{\mathfrak{R}\left(\widehat{g}_{l}\right) \mathfrak{R}\left(g_{l}\right)\right\}=\mathbb{E}\left\{\mathfrak{J}\left(\widehat{g}_{l}\right) \mathfrak{I}\left(g_{l}\right)\right\}=\sigma_{g^{2}}^{2}, \\
& R_{\mathrm{q}}=\mathbb{E}\left\{\mathfrak{R}\left(\widehat{g}_{l}\right) \mathfrak{I}\left(g_{l}\right)\right\}=-\mathbb{E}\left\{\mathfrak{J}\left(\widehat{g}_{l}\right) \mathfrak{R}\left(g_{l}\right)\right\}=0,
\end{aligned}
$$

where $\mathbb{E}\{x\}$ is the expectation of $x, \mathfrak{R}(x)$ is the real part of $x$, and $\mathfrak{J}(x)$ is the imaginary part of $x . u_{l}$ and $v_{l}$ are i.i.d. zero mean Gaussian RVs, which are both independent of $\widehat{g}_{l}$. The variance of $u_{l}$ or $v_{l}$ is $\sigma_{e}^{2}=\sigma_{g}^{2}\left(1-|\rho|^{2}\right)$, where $\rho$ is the complex correlation coefficient between $\widehat{g}_{l}$ and $g_{l}$ [23]:

$$
\rho=\frac{\mathbb{E}\left\{g_{l} \hat{g}_{l}^{*}\right\}}{\sqrt{\mathbb{E}\left\{g_{l} g_{l}^{*}\right\} \mathbb{E}\left\{\hat{g}_{l} \hat{g}_{l}^{*}\right\}}}=\frac{R_{\mathrm{c}}+j R_{\mathrm{q}}}{\sigma_{g} \sigma_{\widehat{g}}}=\frac{\sigma_{g}}{\sigma_{\widehat{g}}} .
$$

From (5), $|\rho|=\rho=\sigma_{g} / \sigma_{\widehat{g}}$. Considering the similarity between PBNJ and AWGN, there is a similar decomposition between $g_{l}$ and $\hat{g}_{l}^{\prime}$, with $\rho^{\prime}=\sigma_{g} / \sigma_{\hat{g}^{\prime}}$ and $\sigma_{e^{\prime}}^{2}=\sigma_{g}^{2}\left(1-\rho^{\prime 2}\right)$. For each single hop, we define the average SNR and the signal to jamming plus noise ratio (SJNR) as

$$
\begin{gathered}
\bar{\gamma}=\frac{\sigma_{g}^{2} P_{\mathrm{d}}}{\sigma_{n}^{2}}, \\
\bar{\gamma}^{\prime}=\frac{\sigma_{g}^{2} P_{\mathrm{d}}}{\sigma_{n}^{2}+\sigma_{J}^{2}} .
\end{gathered}
$$

Considering the influence of channels estimation error, we further define the effective SNR and the effective SJNR as

$$
\begin{gathered}
\bar{\gamma}_{\rho}=\frac{\rho^{2} \bar{\gamma}}{1+\bar{\gamma}\left(1-\rho^{2}\right)}, \\
\bar{\gamma}_{\rho^{\prime}}=\frac{\rho^{\prime 2} \bar{\gamma}^{\prime}}{1+\bar{\gamma}^{\prime}\left(1-\rho^{\prime 2}\right)} .
\end{gathered}
$$

\section{Performance Analysis of EGC Receiver}

In this section, we first derive the BER of FFH/BPSK with EGC receiver, which further simplifies the results obtained in [22]. Then we calculate a closed-form BER expression for the case with $L=2$. 
3.1. BER for an Arbitrary L. In the presence of PBNJ, the EGC output is

$$
r_{\mathrm{EGC}}=\sum_{l=1}^{M} \frac{\hat{g}_{l}^{\prime *}}{\left|\hat{g}_{l}^{\prime}\right|} y_{l}^{\prime}+\sum_{l=M+1}^{L} \frac{\widehat{g}_{l}^{*}}{\left|\widehat{g}_{l}\right|} y_{l},
$$

where $M$ is the number of jammed hops of a symbol.

With the BPSK constellation, the decision statistic is the real part of the combining output. Error occurs with $\mathfrak{R}\left(r_{\mathrm{EGC}}\right)<0$ when $s=\sqrt{P_{\mathrm{d}}}$ is transmitted. Therefore, given $M$ and the set $\mathbf{G}=\left\{g_{1}, \ldots, g_{L}, \widehat{g}_{1}^{\prime}, \ldots, \widehat{g}_{M}^{\prime}, \widehat{g}_{M+1}, \ldots, \widehat{g}_{L}\right\}$, the conditional error probability is

$$
P_{\mathrm{EGC}}(M, \mathbf{G})=\operatorname{Pr}\left(\Re\left(r_{\mathrm{EGC}}\right)<0 \mid s=\sqrt{P_{\mathrm{d}}}, M, \mathbf{G}\right) .
$$

Using (1)-(5), the decision statistic $\mathfrak{R}\left(r_{\mathrm{EGC}}\right)$ is expanded as

$$
\begin{aligned}
\Re\left(r_{\mathrm{EGC}}\right)= & s\left(\rho^{2} \sum_{l=M+1}^{L}\left|\widehat{g}_{l}\right|+\rho^{\prime 2} \sum_{l=1}^{M}\left|\widehat{g}_{l}^{\prime}\right|\right) \\
& +s\left(\sum_{l=M+1}^{L} u_{l}+\sum_{l=1}^{M} u_{l}^{\prime}\right) \\
& +\Re\left(\sum_{l=M+1}^{L} \frac{\widehat{g}_{l}^{*}}{\left|\widehat{g}_{l}\right|} n_{l}+\sum_{l=1}^{M} \frac{\widehat{g}_{l}^{\prime *}}{\left|\widehat{g}_{l}^{\prime}\right|}\left(J_{l}+n_{l}\right)\right) .
\end{aligned}
$$

According to (10), given $s, M$, and $\mathbf{G}, \mathfrak{R}\left(r_{\mathrm{EGC}}\right)$ is conditional Gaussian distributed. Hence, the $P_{\mathrm{EGC}}(M, \mathbf{G})$ of (9) is calculated to be

$$
P_{\mathrm{EGC}}(M, \mathbf{G})=\mathrm{Q}\left(\frac{\mathbb{E}\left(\Re\left(r_{\mathrm{EGC}}\right) \mid s=\sqrt{P_{\mathrm{d}}}, M, \mathbf{G}\right)}{\sqrt{\operatorname{var}\left(\Re\left(r_{\mathrm{EGC}}\right) \mid s=\sqrt{P_{\mathrm{d}}}, M, \mathbf{G}\right)}}\right),
$$

where $\operatorname{var}(x)$ is the variance of $x$ and $\mathrm{Q}(x)$ is the Gaussian $\mathrm{Q}$ function calculated by

$$
\mathrm{Q}(x)=\frac{1}{\pi} \int_{0}^{\pi / 2} \exp \left(-\frac{x^{2}}{2 \sin ^{2} \psi}\right) \mathrm{d} \psi, \quad x>0 .
$$

Using (10) and (11), we simplify $P_{\mathrm{EGC}}(M, \mathbf{G})$ as

$$
P_{\mathrm{EGC}}(M, \mathbf{G})=\mathrm{Q}\left(\alpha V+\beta V^{\prime}\right) \text {, }
$$

where

$$
\begin{gathered}
V=\frac{1}{\sigma_{\widehat{g}}} \sum_{l=M+1}^{L}\left|\widehat{g}_{l}\right|, \quad V^{\prime}=\frac{1}{\sigma_{\widehat{g}^{\prime}}} \sum_{l=1}^{M}\left|\widehat{g}_{l}^{\prime}\right|, \\
\alpha=\frac{\rho}{\lambda}, \quad \beta=\frac{\rho^{\prime}}{\lambda}, \\
\lambda=\sqrt{\frac{(L-M) \rho^{2}}{\bar{\gamma}_{\rho}}+\frac{M \rho^{\prime 2}}{\bar{\gamma}_{\rho^{\prime}}} .}
\end{gathered}
$$

By defining $Z=\alpha V+\beta V^{\prime}$, the characteristic function (CHF) of $Z$ is given by

$$
\begin{aligned}
\varphi_{Z}(t)= & \left(1-\sqrt{\frac{\pi}{2}} \alpha t e^{-\alpha^{2} t^{2} / 2}\left(-j+\operatorname{erfi}\left(\frac{\alpha t}{\sqrt{2}}\right)\right)\right)^{L-M} \\
& \times\left(1-\sqrt{\frac{\pi}{2}} \beta t e^{-\beta^{2} t^{2} / 2}\left(-j+\operatorname{erfi}\left(\frac{\beta t}{\sqrt{2}}\right)\right)\right)^{M},
\end{aligned}
$$

where erfi $(x)$ is the imaginary error function.

After averaging $P_{\mathrm{EGC}}(M, \mathbf{G})$ over the distribution of $Z$, we obtain the $P_{\mathrm{EGC}}(M)$ as

$$
P_{\mathrm{EGC}}(M)=\frac{1}{2 \pi} \int_{-\infty}^{\infty} \int_{0}^{\infty} \mathrm{Q}(z) e^{-j t z} \varphi_{Z}(t) \mathrm{d} z \mathrm{~d} t,
$$

where the internal integration of (16) can be calculated in a closed form, as

$$
\begin{aligned}
\Xi(t)= & \int_{0}^{\infty} \mathrm{Q}(z) e^{-j t z} \mathrm{~d} z \\
= & \frac{1}{\pi} \int_{0}^{\pi / 2} \int_{0}^{\infty} \exp \left(-\frac{z^{2}}{2 \sin ^{2} \psi}\right) e^{-j t z} \mathrm{~d} z \mathrm{~d} \psi \\
= & \frac{1}{\sqrt{2 \pi}} \int_{0}^{\pi / 2} \exp \left(-\frac{t^{2} \sin ^{2} \psi}{2}\right) \\
& \cdot\left(1-j \operatorname{erfi}\left(\frac{t \sin \psi}{\sqrt{2}}\right)\right) \sin \psi \mathrm{d} \psi \\
= & -j \frac{1}{2 t}+\frac{1}{2 t} \exp \left(-\frac{t^{2}}{2}\right)\left(j+\operatorname{erfi}\left(\frac{t}{\sqrt{2}}\right)\right) .
\end{aligned}
$$

Then $P_{\mathrm{EGC}}(M)$ is simplified to be

$$
P_{\mathrm{EGC}}(M)=\frac{1}{2 \pi} \int_{-\infty}^{\infty} \Xi(t) \varphi_{Z}(t) \mathrm{d} t .
$$

Since $\varphi_{Z}(t)$ involves the CHF of a Rayleigh sum, a closed form for $P_{\mathrm{EGC}}(M)$ with an arbitrary $L$ has not yet been available so far as we know [24]. Compared with the quad-slope integration given by [22], we simplify $P_{\mathrm{EGC}}(M)$ to be a 1-tuple integration, which reduces the complexity of numerical calculation.

Finally, the average error probability of EGC receiver is

$$
P_{\mathrm{EGC}}=\sum_{M=0}^{L}\left(\begin{array}{c}
L \\
M
\end{array}\right) \rho_{\mathrm{PBNJ}}^{M}\left(1-\rho_{\mathrm{PBNJ}}\right)^{L-M} P_{\mathrm{EGC}}(M) \text {. }
$$

3.2. Closed-Form BER Expression with $L=2$. In the special case with $L=2$, we work out a closed-form expression for $P_{\mathrm{EGC}}(M)$. When $M=0, P_{\mathrm{EGC}}(M=0)$ is given by [25]

$$
P_{\mathrm{EGC}}(M=0)=\frac{1}{2}\left(1-\frac{\sqrt{\bar{\gamma}_{\rho}\left(2+\bar{\gamma}_{\rho}\right)}}{1+\bar{\gamma}_{\rho}}\right) \text {. }
$$

Similarly, $P_{\mathrm{EGC}}(M=2)$ is calculated to be

$$
P_{\mathrm{EGC}}(M=2)=\frac{1}{2}\left(1-\frac{\sqrt{\bar{\gamma}_{\rho^{\prime}}\left(2+\bar{\gamma}_{\rho^{\prime}}\right)}}{1+\bar{\gamma}_{\rho^{\prime}}}\right) \text {. }
$$


For the case with $M=1$, we have $V \sim \operatorname{Rayleigh}(1), V^{\prime} \sim$ $\operatorname{Rayleigh}(1)$, and $\lambda=\sqrt{\rho^{2} / \bar{\gamma}_{\rho}+\rho^{\prime 2} / \bar{\gamma}_{\rho^{\prime}}}$. Then $P_{\mathrm{EGC}}(M=1)$ is calculated by

$$
P_{\mathrm{EGC}}(M=1)=\int_{0}^{\infty} \int_{0}^{\infty} \mathrm{Q}(\alpha x+\beta y) f_{V}(x) f_{V^{\prime}}(y) \mathrm{d} x \mathrm{~d} y,
$$

which can be further expanded with (12), as

$$
\begin{aligned}
& P_{\mathrm{EGC}}(M=1) \\
& =\frac{1}{\pi} \int_{0}^{\pi / 2} \int_{0}^{\infty} \int_{0}^{\infty} \exp \left(-\frac{(\alpha x+\beta y)^{2}}{2 \sin ^{2} \theta}\right) f_{V}(x) \\
& \cdot f_{V^{\prime}}(y) \mathrm{d} x \mathrm{~d} y \mathrm{~d} \theta .
\end{aligned}
$$

We first solve the internal integral with $x$, as

$$
\begin{aligned}
& A(y, \theta)=\int_{0}^{\infty} \exp \left(-\frac{(\alpha x+\beta y)^{2}}{2 \sin ^{2} \theta}\right) f_{V}(x) f_{V^{\prime}}(y) \mathrm{d} x \\
& =y\left(1+\alpha^{2} \csc ^{2} \theta\right)^{3 / 2} e^{-y^{2}\left(1+\beta^{2} \csc ^{2} \theta\right) / 2} \\
& \times\left(\sqrt{1+\alpha^{2} \csc ^{2} \theta}\right. \\
& -\sqrt{2 \pi} y \alpha \beta \csc ^{2} \theta e^{y^{2} \alpha^{2} \beta^{2} \csc ^{4} \theta /\left(2+2 \alpha^{2} \csc ^{2} \theta\right)} \\
& \left.\mathrm{Q}\left(\frac{y \alpha \beta \csc ^{2} \theta}{\sqrt{1+\alpha^{2} \csc ^{2} \theta}}\right)\right) \text {. }
\end{aligned}
$$

Then we solve the internal integral with $y$, as

$$
\begin{aligned}
& B(\theta)=\int_{0}^{\infty} A(y, \theta) \mathrm{d} y \\
& =\left(1+\frac{2 \beta^{2}}{1+2 \alpha^{2}-\cos 2 \theta}\right)^{-3 / 2}\left(1+\alpha^{2} \csc ^{2} \theta\right)^{-5 / 2} \\
& \cdot\left(1+\beta^{2} \csc ^{2} \theta\right)^{-1}\left(\frac{\csc \theta^{2}}{\alpha^{2}+\sin ^{2} \theta}\right)^{-1 / 2} \\
& \times\left\{\alpha^{2} \beta^{2} \sqrt{1+\frac{2 \beta^{2}}{1+2 \alpha^{2}-\cos 2 \theta}} \csc ^{6} \theta\right. \\
& -\sqrt{\frac{2 \csc ^{2} \theta}{1+2 \alpha^{2}-\cos 2 \theta}} \\
& \times\left[\alpha \beta \arctan \frac{\sqrt{1+2 \beta^{2} /\left(1+2 \alpha^{2}-\cos 2 \theta\right)}}{\alpha \beta \sqrt{\csc \theta^{2} /\left(\alpha^{2}+\sin \theta^{2}\right)}}\right. \\
& \cdot \csc ^{2} \theta\left(1+\alpha^{2} \csc ^{2} \theta\right)\left(1+\beta^{2} \csc ^{2} \theta\right)
\end{aligned}
$$

$$
\begin{aligned}
& -\sqrt{1+\frac{2 \beta^{2}}{1+2 \alpha^{2}-\cos 2 \theta}} \\
& \left.\left.\cdot \sqrt{1+\alpha^{2} \csc ^{2} \theta}\left(1+\left(\alpha^{2}+\beta^{2}\right) \csc ^{2} \theta\right)\right]\right\} .
\end{aligned}
$$

Finally, we calculate the internal integral with $\theta$, as

$$
\begin{aligned}
P_{\mathrm{EGC}}(M=1) & =\frac{1}{\pi} \int_{0}^{\pi / 2} B(\theta) \mathrm{d} \theta \\
& =\frac{1}{2}\left(1-\frac{\alpha \sqrt{1+\alpha^{2}}+\beta \sqrt{1+\beta^{2}}}{1+\alpha^{2}+\beta^{2}}\right) .
\end{aligned}
$$

By substituting (20), (21), and (26) into (19), we obtain the closed-form BER expression for $L=2$.

\section{Adaptive Jamming Suppression Schemes}

4.1. $W$-EGC Receiver. In the $\mathrm{W}$-EGC receiver, the jammed and unjammed received signal are, respectively, sent into an EGC receiver. Then the two EGC outputs are weighted and combined, with final output as

$$
r_{\mathrm{W}-\mathrm{EGC}}=\eta \sum_{l=1}^{M} \frac{\hat{g}_{l}^{\prime *}}{\left|\widehat{g}_{l}^{\prime}\right|} y_{l}^{\prime}+\sum_{l=M+1}^{L} \frac{\widehat{g}_{l}^{*}}{\left|\widehat{g}_{l}\right|} y_{l}
$$

where $\eta$ is the weighting coefficient. In the following analysis, we will optimize $\eta$ to minimize the BER.

Similar to (13), the conditional error probability of the WEGC receiver can be written as

$$
P_{\mathrm{W}-\mathrm{EGC}}(M, \mathbf{G})=\mathrm{Q}\left(a V+\eta b V^{\prime}\right),
$$

where

$$
\begin{gathered}
a=\frac{\rho}{\mu}, \quad b=\frac{\rho^{\prime}}{\mu}, \\
\mu=\sqrt{\frac{(L-M) \rho^{2}}{\bar{\gamma}_{\rho}}+\frac{\eta^{2} M \rho^{\prime 2}}{\bar{\gamma}_{\rho^{\prime}}}} .
\end{gathered}
$$

Note that the Gaussian Q function is a monotone decreasing function; that is, minimizing $P_{\mathrm{W} \text {-EGC }}$ is equivalent to maximizing $a V+\eta b V^{\prime}$. By solving $\partial\left(a V+\eta b V^{\prime}\right) / \partial \eta=0$, the optimum $\eta$ can be obtained as

$$
\eta_{\mathrm{opt}}=\frac{L-M}{M} \frac{\bar{\gamma}_{\rho^{\prime}}}{\bar{\gamma}_{\rho}} \frac{\sum_{l=1}^{M}\left|\widehat{g}_{l}^{\prime}\right|}{\sum_{l=M+1}^{L}\left|\hat{g}_{l}\right|} .
$$

After substituting (30) into (28), we simplify $P_{\mathrm{W}-\mathrm{EGC}}$ as

$$
P_{\mathrm{W}-\mathrm{EGC}}=\mathrm{Q}\left(\sqrt{c_{1} V^{2}+c_{2} V^{\prime 2}}\right) \text {, }
$$


where $c_{1}=\bar{\gamma}_{\rho} /(L-M)$ and $c_{2}=\bar{\gamma}_{\rho^{\prime}} / M$. Once again, we use the CHF method and calculate the $P_{\mathrm{W}-\mathrm{EGC}}$ as

$$
\begin{gathered}
P_{\mathrm{W}-\mathrm{EGC}} \\
\begin{array}{c}
\frac{1}{4 \pi^{2}} \int_{0}^{\infty} \int_{0}^{\infty} \int_{-\infty}^{\infty} \int_{-\infty}^{\infty} \mathrm{Q}\left(\sqrt{c_{1} x^{2}+c_{2} y^{2}}\right) \\
\cdot e^{-j t_{1} x} e^{-j t_{2} y} \varphi_{V}\left(t_{1}\right) \\
\cdot \varphi_{V^{\prime}}\left(t_{2}\right) \mathrm{d} t_{1} \mathrm{~d} t_{2} \mathrm{~d} x \mathrm{~d} y \\
=\frac{1}{4 \pi^{2}} \int_{0}^{\pi / 2} \int_{0}^{\infty} \int_{0}^{\infty} \int_{-\infty}^{\infty} \int_{-\infty}^{\infty} \frac{1}{\pi} \exp \left(-\frac{c_{1} x^{2}+c_{2} y^{2}}{2 \sin ^{2} \psi}\right) \\
\cdot e^{-j\left(t_{1} x+t_{2} y\right)} \varphi_{V}\left(t_{1}\right) \\
\cdot \varphi_{V^{\prime}}\left(t_{2}\right) \mathrm{d} t_{1} \mathrm{~d} t_{2} \mathrm{~d} x \mathrm{~d} y \\
=\frac{1}{4 \pi^{2}} \int_{0}^{\pi / 2} \int_{-\infty}^{\infty} \int_{-\infty}^{\infty} f\left(t_{1}, t_{2}, \psi\right) \\
\varphi_{V}\left(t_{1}\right) \varphi_{V^{\prime}}\left(t_{2}\right) \mathrm{d} t_{1} \mathrm{~d} t_{2} \mathrm{~d} \psi,
\end{array}
\end{gathered}
$$

where

$$
\begin{aligned}
& f\left(t_{1}, t_{2}, \psi\right)=\frac{1}{\pi}\left(\int_{0}^{\infty} \exp \left(-\frac{c_{1} x^{2}}{2 \sin ^{2} \psi}\right) e^{-j t_{1} x} \mathrm{~d} x\right) \\
& \cdot\left(\int_{0}^{\infty} \exp \left(-\frac{c_{2} y^{2}}{2 \sin ^{2} \psi}\right) e^{-j t_{2} y} \mathrm{~d} y\right) \\
& =-\frac{\sin ^{2} \psi}{2 \sqrt{c_{1} c_{2}}} \exp \left(-\frac{1}{2}\left(\frac{t_{1}^{2}}{c_{1}}+\frac{t_{2}^{2}}{c_{2}}\right) \sin ^{2} \psi\right) \\
& \cdot\left(j+\operatorname{erfi}\left(\frac{t_{1} \sin \psi}{\sqrt{2 c_{1}}}\right)\right) \\
& \cdot\left(j+\operatorname{erfi}\left(\frac{t_{2} \sin \psi}{\sqrt{2 c_{2}}}\right)\right), \\
& \varphi_{V}\left(t_{1}\right)=\left(1-e^{-t_{1}^{2} / 2} \sqrt{\frac{\pi}{2}} t_{1}\left(-j+\operatorname{erfi}\left(\frac{t_{1}}{\sqrt{2}}\right)\right)\right)^{L-M}, \\
& \varphi_{V^{\prime}}\left(t_{2}\right)=\left(1-e^{-t_{2}^{2} / 2} \sqrt{\frac{\pi}{2}} t_{2}\left(-j+\operatorname{erfi}\left(\frac{t_{2}}{\sqrt{2}}\right)\right)\right)^{M} .
\end{aligned}
$$

Note that $\eta$ is eliminated in (28) with $M=0$ or $M=L$, which indicates that $\eta_{\text {opt }}$ is used only for $1 \leq M \leq L-1$. Due to the Rayleigh sum involved, a closed-form expression for $P_{\text {W-EGC }}$ is not available for an arbitrary $L$. Similar to (19), the BER for W-EGC is

$$
P_{\mathrm{W}-\mathrm{EGC}}=\sum_{M=0}^{L}\left(\begin{array}{c}
L \\
M
\end{array}\right) \rho_{\mathrm{PBNJ}}^{M}\left(1-\rho_{\mathrm{PBNJ}}\right)^{L-M} P_{\mathrm{W}-\mathrm{EGC}}(M) .
$$

We would like to compare the BER between EGC and WEGC. Due to the monotonicity of the Gaussian Q function, we only need to compare the internal fraction of (13) and (31), which is calculated to be

$$
\begin{aligned}
\vartheta\left(V, V^{\prime}\right)= & c_{1} V^{2}+c_{2} V^{\prime 2}-\left(\alpha V+\beta V^{\prime}\right)^{2} \\
= & \frac{M}{L-M} \frac{\rho^{2}}{(L-M) \rho^{2} \bar{\gamma}_{\rho^{\prime}}+M \rho^{\prime 2} \bar{\gamma}_{\rho}} \\
& \cdot\left(\frac{\rho^{\prime}}{\rho} \bar{\gamma}_{\rho} V-\frac{L-M}{M} \bar{\gamma}_{\rho^{\prime}} V^{\prime}\right)^{2} .
\end{aligned}
$$

Take the expectation of $\vartheta\left(V, V^{\prime}\right)$ with regard to $V$ and $V^{\prime}$, as

$$
\begin{aligned}
\mathbb{E}\left\{\vartheta\left(V, V^{\prime}\right)\right\}= & \frac{M}{(L-M) \bar{\gamma}_{\rho^{\prime}}+\left(\rho^{\prime 2} / \rho^{2}\right) M \bar{\gamma}_{\rho}} \\
& \cdot\left(\left(2-\frac{\pi}{2}\right)\left[\left(\frac{L}{M}-1\right) \bar{\gamma}_{\rho^{\prime}}^{2}+\bar{\gamma}_{\rho}^{2} \frac{\rho^{\prime 4}}{\rho^{4}}\right]\right. \\
& \left.+\frac{\pi}{2}(L-M)\left(\bar{\gamma}_{\rho^{\prime}}-\bar{\gamma}_{\rho} \frac{\rho^{\prime 2}}{\rho^{2}}\right)^{2}\right)>0 .
\end{aligned}
$$

From (36), it is shown that, as compared with EGC, W-EGC has a lower conditional error probability. Besides, with the increase of SJR, we have

$$
\lim _{\sigma_{J}^{2} \rightarrow 0} \mathbb{E}\left\{\vartheta\left(V, V^{\prime}\right)\right\}=\left(2-\frac{\pi}{2}\right) \bar{\gamma}_{\rho}>0
$$

From (37), it is indicated that, in a high SJR region, W-EGC will lower the error floor, which is determined by the SNR.

In the special case with $L=2, P_{\mathrm{W}-\mathrm{EGC}}$ has a closed-form expression. For $M=0$ and $M=2$, we have $P_{\mathrm{W}-\mathrm{EGC}}(M=$ $0,2)=P_{\mathrm{EGC}}(M=0,2)$. For $M=1, P_{\mathrm{W}-\mathrm{EGC}}(M=1)$ is calculated to be

$$
\begin{aligned}
& P_{\mathrm{W}-\mathrm{EGC}}(M=1) \\
& =\int_{0}^{\infty} \int_{0}^{\infty} \mathrm{Q}\left(\sqrt{\bar{\gamma}_{\rho} x+\bar{\gamma}_{\rho^{\prime}} y}\right) f_{X}(x) f_{Y}(y) \mathrm{d} x \mathrm{~d} y \\
& =\frac{1}{4 \pi} \int_{0}^{\pi / 2} \int_{0}^{\infty} \int_{0}^{\infty} \exp \left(-\frac{\bar{\gamma}_{\rho} x+\bar{\gamma}_{\rho^{\prime}} y}{2 \sin ^{2} \psi}-\frac{x+y}{2}\right) \mathrm{d} x \mathrm{~d} y \mathrm{~d} \psi \\
& =\frac{1}{\pi} \int_{0}^{\pi / 2} \frac{1}{\left(1+\bar{\gamma}_{\rho} \csc ^{2} \psi\right)\left(1+\bar{\gamma}_{\rho^{\prime}} \csc ^{2} \psi\right)} \mathrm{d} \psi \\
& =\frac{1}{2}\left(1-\sqrt{\frac{\bar{\gamma}_{\rho^{\prime}}}{1+\bar{\gamma}_{\rho^{\prime}}}} \frac{\bar{\gamma}_{\rho^{\prime}}}{\bar{\gamma}_{\rho^{\prime}}-\bar{\gamma}_{\rho}}+\sqrt{\frac{\bar{\gamma}_{\rho}}{1+\bar{\gamma}_{\rho}}} \frac{\bar{\gamma}_{\rho}}{\bar{\gamma}_{\rho^{\prime}}-\bar{\gamma}_{\rho}}\right) .
\end{aligned}
$$

4.2. Suboptimum W-EGC Receiver. Note that the optimum weighting coefficient of (30) contains the instantaneous channel estimates. To achieve a simpler result, we replace 
the $\left|\widehat{g}_{l}\right|$ and $\left|\hat{g}_{l}^{\prime}\right|$ with the corresponding mathematical expectations $\sqrt{\pi / 2} \sigma_{\widehat{g}}$ and $\sqrt{\pi / 2} \sigma_{\widehat{g}^{\prime}}$, respectively. The suboptimum weighting coefficient $\eta_{\text {sopt }}$ is then calculated to be

$$
\eta_{\text {sopt }}=\frac{\bar{\gamma}_{\rho^{\prime}}}{\rho^{\prime} \bar{\gamma}_{\rho}} .
$$

The conditional error probability of the suboptimum WEGC (SW-EGC) receiver is calculated to be

$$
P_{\text {SW-EGC }}(M, \mathbf{G})=\mathrm{Q}\left(\alpha_{\mathrm{S}} V+\beta_{\mathrm{S}} V^{\prime}\right) \text {, }
$$

where

$$
\begin{gathered}
\alpha_{\mathrm{S}}=\frac{\rho \bar{\gamma}_{\rho}}{\lambda_{\mathrm{S}}}, \quad \beta_{\mathrm{S}}=\frac{\bar{\gamma}_{\rho^{\prime}}}{\lambda_{\mathrm{S}}}, \\
\lambda_{\mathrm{S}}=\sqrt{\rho^{2}(L-M) \bar{\gamma}_{\rho}+M \bar{\gamma}_{\rho^{\prime}}} .
\end{gathered}
$$

Since $P_{\text {SW-EGC }}(M, \mathbf{G})$ and $P_{\text {EGC }}(M, \mathbf{G})$ are in similar form, $P_{\text {SW-EGC }}(M)$ can be simply obtained by substituting (41) into (18). Similarly, the closed-form expressions for $P_{\text {SW-EGC }}(M, \mathbf{G})$ with $L=2$ are given by $P_{\text {SW-EGC }}(M=0,2)=$ $P_{\mathrm{EGC}}(M=0,2)$ and

$$
P_{\mathrm{SW}-\mathrm{EGC}}(M=1)=\frac{1}{2}\left(1-\frac{\alpha_{\mathrm{S}} \sqrt{1+\alpha_{\mathrm{S}}^{2}}+\beta_{\mathrm{S}} \sqrt{1+\beta_{\mathrm{S}}^{2}}}{1+\alpha_{\mathrm{S}}^{2}+\beta_{\mathrm{S}}^{2}}\right) .
$$

Similar to (35), the comparison between the SW-EGC and EGC can be denoted as $\vartheta_{\mathrm{S}}\left(V, V^{\prime}\right)=\left(\alpha_{\mathrm{S}}-\alpha\right) V+\left(\beta_{\mathrm{S}}-\beta\right) V^{\prime}$. In the high SJR region, we have

$$
\lim _{\sigma_{J}^{2} \rightarrow 0} \mathbb{E}\left\{\vartheta_{S}\left(V, V^{\prime}\right)\right\}=\left(\frac{\rho(L-M)+M}{\sqrt{\rho^{2}(L-M)+M}}-\sqrt{L}\right) \sqrt{\frac{\pi}{2} \bar{\gamma}_{\rho}} .
$$

For a moderate large SNR, that is, $\rho^{2} \rightarrow 1$, we can see

$$
\lim _{\sigma_{J}^{2} \rightarrow 0, \rho^{2} \rightarrow 1} \mathbb{E}\left\{\vartheta_{S}\left(V, V^{\prime}\right)\right\}=0
$$

From (44), it is indicated that, with a moderate large SNR, the SW-EGC and EGC receivers approach exactly the same error floor.

\section{Numerical Results and Discussions}

In this section, we present some numerical results and corresponding discussions. In simulation, the channel coefficient for each frequency and the location of jammed bandwidth are assumed to be unchanged within a frame. The simulation parameters are given in Table 1.

In Figure 1, the analytical results match the simulation very well. Both W-EGC and SW-EGC outperform EGC in terms of BER, especially in the low $E_{\mathrm{b}} / J_{0}$ region. When
TABLE 1: Simulation parameters.

\begin{tabular}{lc}
\hline Type & Value \\
\hline Frequency subset size & 15 \\
Frame length & $0.9 \mathrm{~ms}$ \\
Hopping rate & $1 \times 10^{5}$ hops/s \\
Number of pilot groups & 2 \\
Pilot power $P_{\mathrm{p}}$ & $P_{\mathrm{p}}=P_{\mathrm{d}}$ \\
Fading type & Frequency selective Rayleigh fading \\
$2 \sigma_{g}^{2}$ & 1 \\
Jamming type & PBNJ \\
Channel estimation method & Pilot-based LS estimation \\
\hline
\end{tabular}

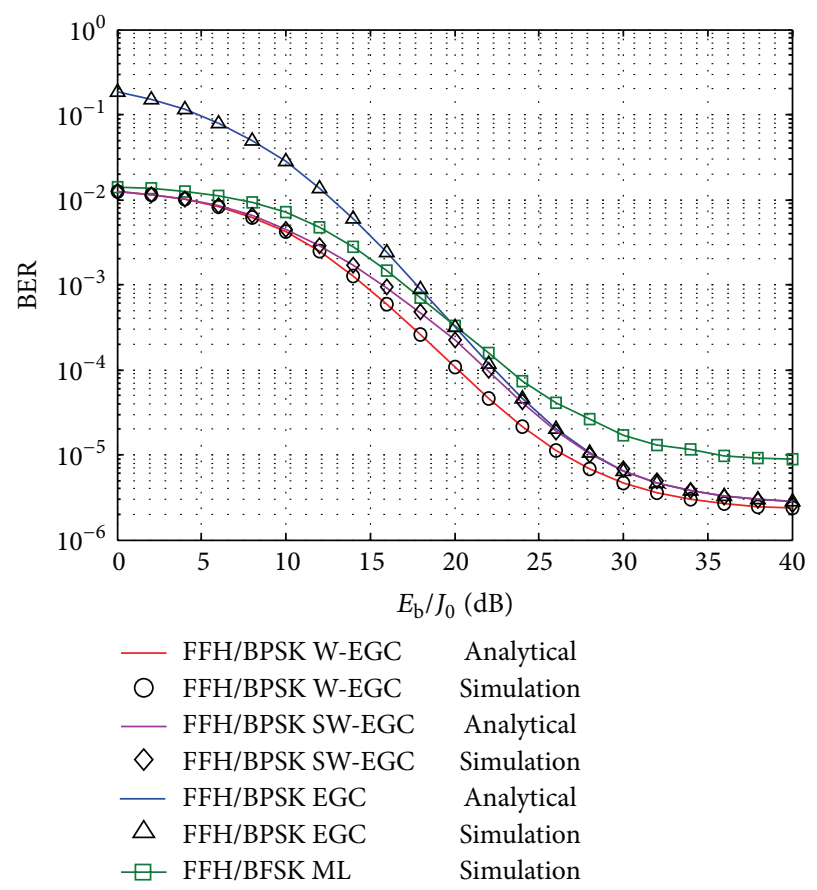

FIGURE 1: BER comparison, with $E_{\mathrm{b}} / N_{0}=25 \mathrm{~dB}, L=3$, and $\rho_{\text {PBNJ }}=$ 0.3 .

BER $=1 \times 10^{-3}$, for example, W-EGC and SW-EGC show, respectively, $3.5 \mathrm{~dB}$ and $2 \mathrm{~dB}$ gain over EGC. In a high $E_{\mathrm{b}} / J_{0}$ region, W-EGC shows a lower error floor than that of EGC, while the SW-EGC receiver approaches the same error floor, which have been explained in Section 4. As compared to the noncoherent FFH/BFSK with ML receiver, which is the optimum receiver for the noncoherent $\mathrm{FFH}$ in the presence of jamming, coherent FFH/BPSK with W-EGC and SW-EGC receivers shows performance gain even with imperfect CSI. As seen in Figure 1, the performance gain is $2.5 \mathrm{~dB}$ and $1 \mathrm{~dB}$, respectively, when $\mathrm{BER}=1 \times 10^{-3}$. And this performance improvement increases with the increase of $E_{\mathrm{b}} / J_{0}$.

Figure 2 shows the influence of the jamming factor $\rho_{\text {PBNJ }}$ on the performance of EGC, W-EGC, and SW-EGC, respectively. For the EGC receiver, there is a worst case jamming factor, which is less than 1 . With the worst case jamming factor, the hostile jammer achieves the worst case jamming effect by jamming only a small fraction of the bandwidth. In 


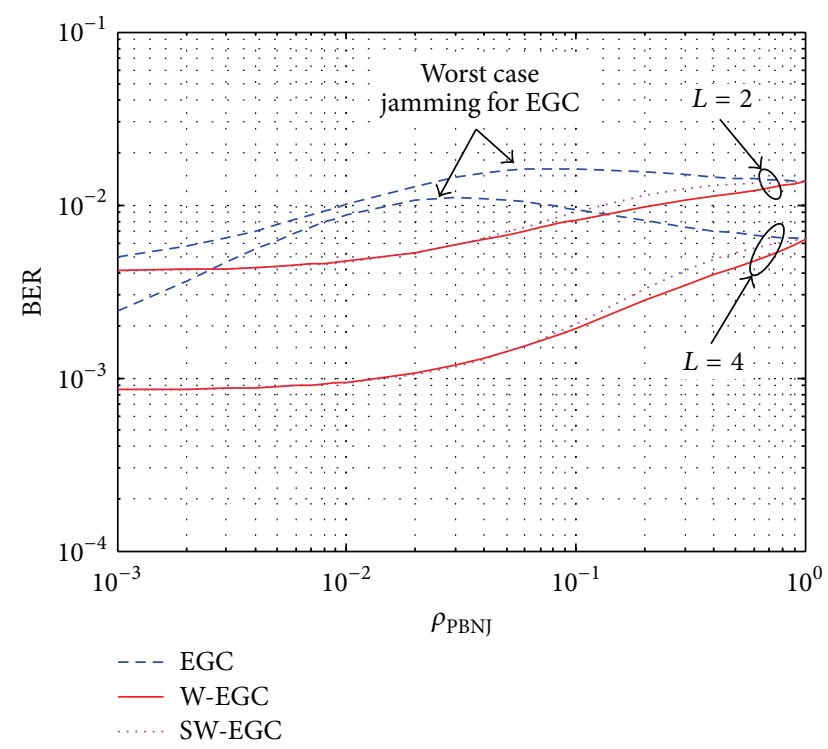

FIGURE 2: BER of FFH/BPSK with W-EGC and EGC versus $\rho_{\text {PBNJ }}$ for $L=2$ and $4, E_{\mathrm{b}} / N_{0}=15 \mathrm{~dB}$, and $E_{\mathrm{b}} / J_{0}=15 \mathrm{~dB}$.

contrast, as seen in Figure 2, the worst case jamming factor for the W-EGC and SW-EGC receiver is 1 . Then the jammer has to take full-band jamming to achieve the worst case jamming, whose jamming effectiveness is greatly reduced.

\section{Conclusion}

We have proposed jamming suppression schemes for coherent FFH/BPSK system, which is based on the weighted equal gain combining. And we analyzed the BER performance in the presence of PBNJ, frequency selective Rayleigh fading channel, and imperfect CSI. From theoretical analysis and simulation validation, it is shown that the proposed schemes significantly outperform EGC and noncoherent ML receiver in terms of BER. Besides, the proposed schemes greatly reduce the jammer's efficiency, where the jammer has to implement full-band jamming to achieve the worst case jamming.

\section{Conflict of Interests}

The authors declare that there is no conflict of interests regarding the publication of this paper.

\section{Acknowledgments}

This work is supported in part by Natural Science Foundation of China (NSFC) Grant no. 61201126, Ministry Sponsored Project 4010103020201-2, Program for New Century Excellent Talents in University (no. NCET-11-0058), and Program for Sichuan Youth Science and Technology Fund (no. 2012JQ0020).

\section{References}

[1] L.-M.-D. Le, K. C. Teh, and K. H. Li, "Jamming rejection using FFH/MFSK ML receiver over fading channels with the presence of timing and frequency offsets," IEEE Transactions on Information Forensics and Security, vol. 8, no. 7, pp. 1195-1200, 2013.

[2] F. Yang and L.-L. Yang, "A single-user noncoherent combining scheme achieving multiuser interference mitigation for FFH/MFSK systems," IEEE Transactions on Wireless Communications, vol. 12, no. 9, pp. 4306-4314, 2013.

[3] G. Li, Q. Wang, V. K. Bhargava, and L. J. Mason, "Maximumlikelihood diversity combining in partial-band noise," IEEE Transactions on Communications, vol. 46, no. 12, pp. 1569-1574, 1998.

[4] J. Zhang, K. C. Teh, and K. H. Li, "Performance analysis of a maximum-likelihood FFH/MFSK receiver with partial-bandnoise jamming over frequency-selective fading channels," IEEE Communications Letters, vol. 12, no. 6, pp. 401-403, 2008.

[5] J. Zhang, K. C. Teh, and K. H. Li, "Error probability analysis of FFH/MFSK receivers over frequency-selective Rician-fading channels with partial-band-noise jamming," IEEE Transactions on Communications, vol. 57, no. 10, pp. 2880-2885, 2009.

[6] J. Zhang, K. C. Teh, and K. H. Li, "Maximum-likelihood FFH/MFSK receiver over rayleigh-fading channels with composite effects of MTJ and PBNJ," IEEE Transactions on Communications, vol. 59, no. 3, pp. 675-679, 2011.

[7] L.-M. Le, K. C. Teh, and K. H. Li, "Performance analysis of a suboptimum fast frequency-hopped/M-ary frequencyshift-keying maximum-likelihood receiver over Rician-fading channels with composite effects of partial-band noise jamming and multitone jamming," IET Communications, vol. 6, no. 13, pp. 1903-1911, 2012.

[8] K. C. Teh, A. Kot, and K. Li, "Partial-band jammer suppression in FFH spread-spectrum system using FFT," IEEE Transactions on Vehicular Technology, vol. 48, no. 2, pp. 478-486, 1999.

[9] R. Robertson and K. Y. Lee, "Performance of fast frequencyhopped $\mathrm{mfsk}$ receivers with linear and self-normalization combining in a rician fading channel with partial-band interference," IEEE Journal on Selected Areas in Communications, vol. 10, no. 4, pp. 731-741, 1992.

[10] R. Robertson and T. Ha, "Error probabilities of fast frequencyhopped fsk with self-normalization combining in a fading channel with partial-band interference," IEEE Journal on Selected Areas in Communications, vol. 10, no. 4, pp. 714-723, 1992.

[11] C.-D. Chung and P.-C. Huang, "Effects of fading and partialband noise jamming on a fast FH/BFSK acquisition receiver with noise-normalization combination," IEEE Transactions on Communications, vol. 44, no. 1, pp. 94-104, 1996.

[12] K. C. Teh, A. C. Kot, and K. H. Li, "Partial-band jamming rejection of FFH/BFSK with product combining receiver over a Rayleigh-Fading channel," IEEE Communications Letters, vol. 1, no. 3, pp. 64-66, 1997.

[13] T. C. Lim, W. He, and K. H. Li, "Rejection of partial-band noise jamming with $\mathrm{FFH} / \mathrm{BFSK}$ product combining receiver over Nakagami-fading channel," Electronics Letters, vol. 34, no. 10, pp. 960-961, 1998.

[14] G. Huo and M.-S. Alouini, "Another look at the BER performance of FFH/BFSK with product combining over partialband jammed Rayleigh-fading channels," IEEE Transactions on Vehicular Technology, vol. 50, no. 5, pp. 1203-1215, 2001. 
[15] C.-L. Chang and T.-M. Tu, "Performance analysis of FFH/BFSK product-combining receiver with partial-band jamming over independent rician fading channels," IEEE Transactions on Wireless Communications, vol. 4, no. 6, pp. 2629-2635, 2005.

[16] S. Ahmed, L.-L. Yang, and L. Hanzo, "Mellin-transformbased performance analysis of FFH $M$-ary FSK using product combining for combatting partial-band noise jamming," IEEE Transactions on Vehicular Technology, vol. 57, no. 5, pp. 27572765, 2008.

[17] J. Zhang, K. C. Teh, and K. H. Li, "Performance study of fast frequency-hopped/M-ary frequency-shift keying systems with timing and frequency offsets over Rician-fading channels with both multitone jamming and partial-band noise jamming," IET Communications, vol. 4, no. 10, pp. 1153-1163, 2010.

[18] C. M. Keller and M. B. Pursley, "Clipped diversity combining for channels with partial-band interference-part I: clipped-linear combining," IEEE Transactions on Communications, vol. 35, no. 12, pp. 1320-1328, 1988.

[19] L. Xiao, X. Xu, and Y. Yao, "Adaptive threshold clipper combining receiver for fast frequency hopping systems during partialband noise jamming," Tsinghua Science \& Technology, vol. 6, no. 1, pp. 42-44, 2001.

[20] J. J. Kang and K. Teh, "Performance of coherent fast frequencyhopped spread-spectrum receivers with partial-band noise jamming and AWGN," IEE Proceedings: Communications, vol. 152, no. 5, pp. 679-685, 2005.

[21] Y. He, Y. Cheng, Y. Yang, G. Wu, B. Dong, and S. Li, "A subsetbased coherent FFH system," IEEE Communications Letters, vol. 19, no. 2, pp. 199-202, 2015.

[22] Y. He, Y. Cheng, G. Wu, and S. Li, "Performance analysis of FFH/BPSK system with partial band noise jamming and channel estimation error in high-mobility wireless communication scenarios," Chinese Science Bulletin, vol. 59, no. 35, pp. 50115018, 2014.

[23] R. Annavajjala and L. B. Milstein, "Performance analysis of linear diversity-combining schemes on Rayleigh fading channels with binary signaling and Gaussian weighting errors," IEEE Transactions on Wireless Communications, vol. 4, no. 5, pp. 2267-2278, 2005.

[24] J. Hu and N. C. Beaulieu, "Accurate simple closed-form approximations to rayleigh sum distributions and densities," IEEE Communications Letters, vol. 9, no. 2, pp. 109-111, 2005.

[25] A. Annamalai, C. Tellambura, and V. K. Bhargava, "Equalgain diversity receiver performance in wireless channels," IEEE Transactions on Communications, vol. 48, no. 10, pp. 1732-1745, 2000. 

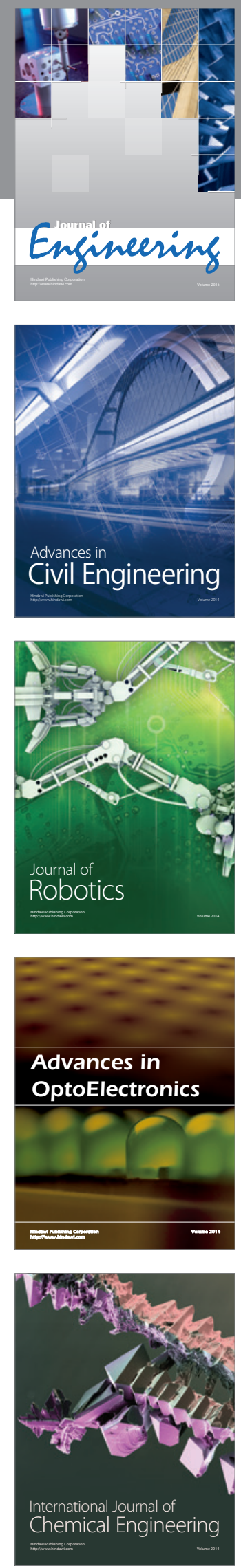

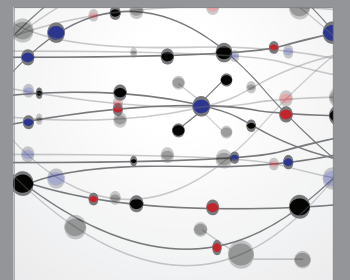

The Scientific World Journal
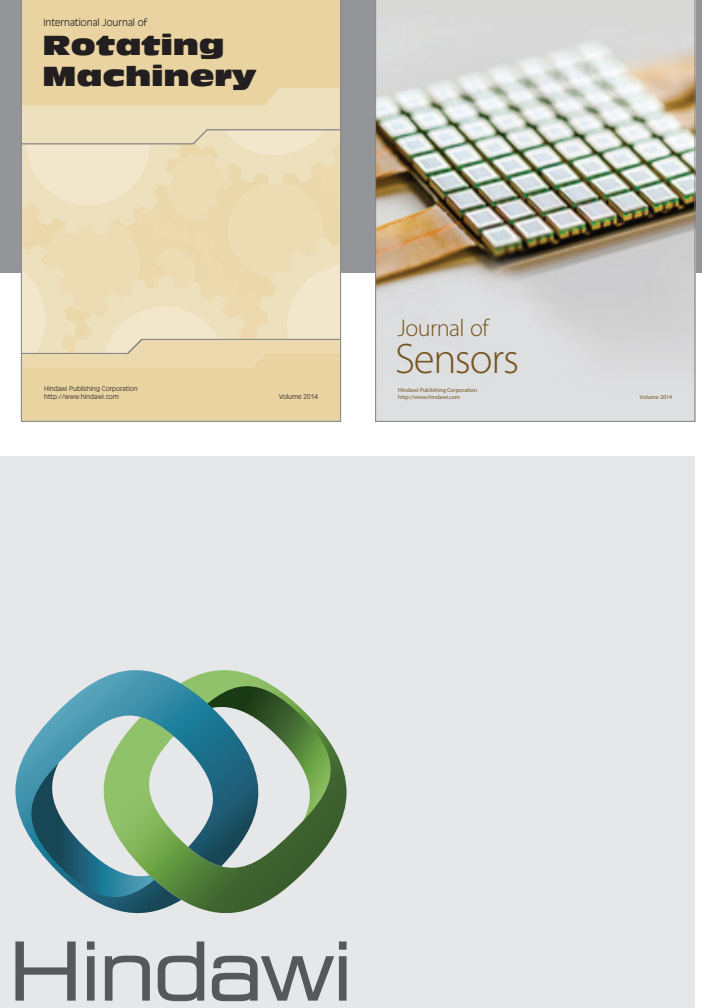

Submit your manuscripts at http://www.hindawi.com
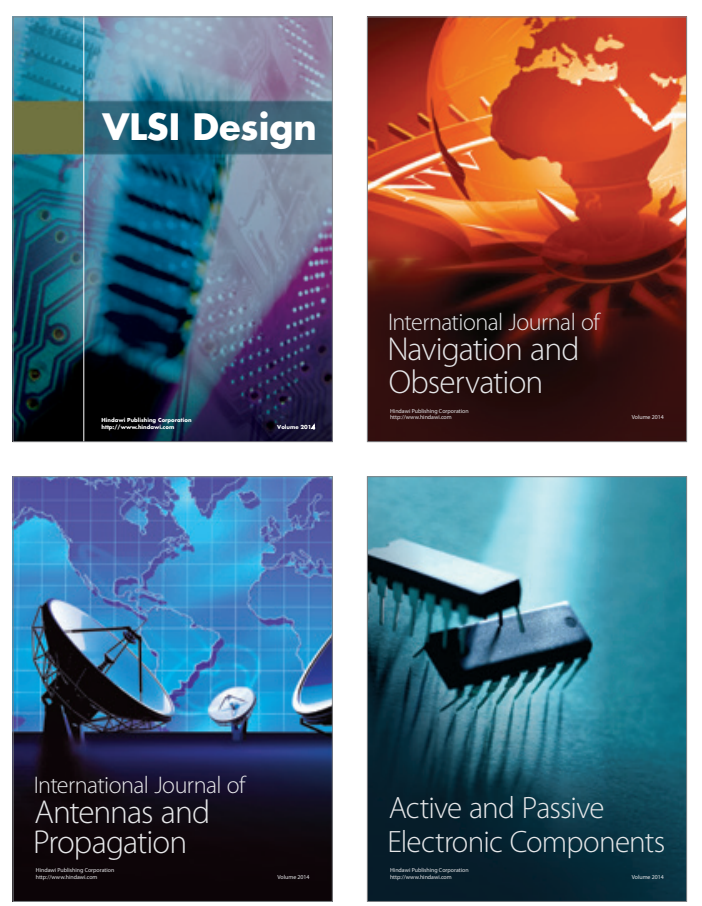
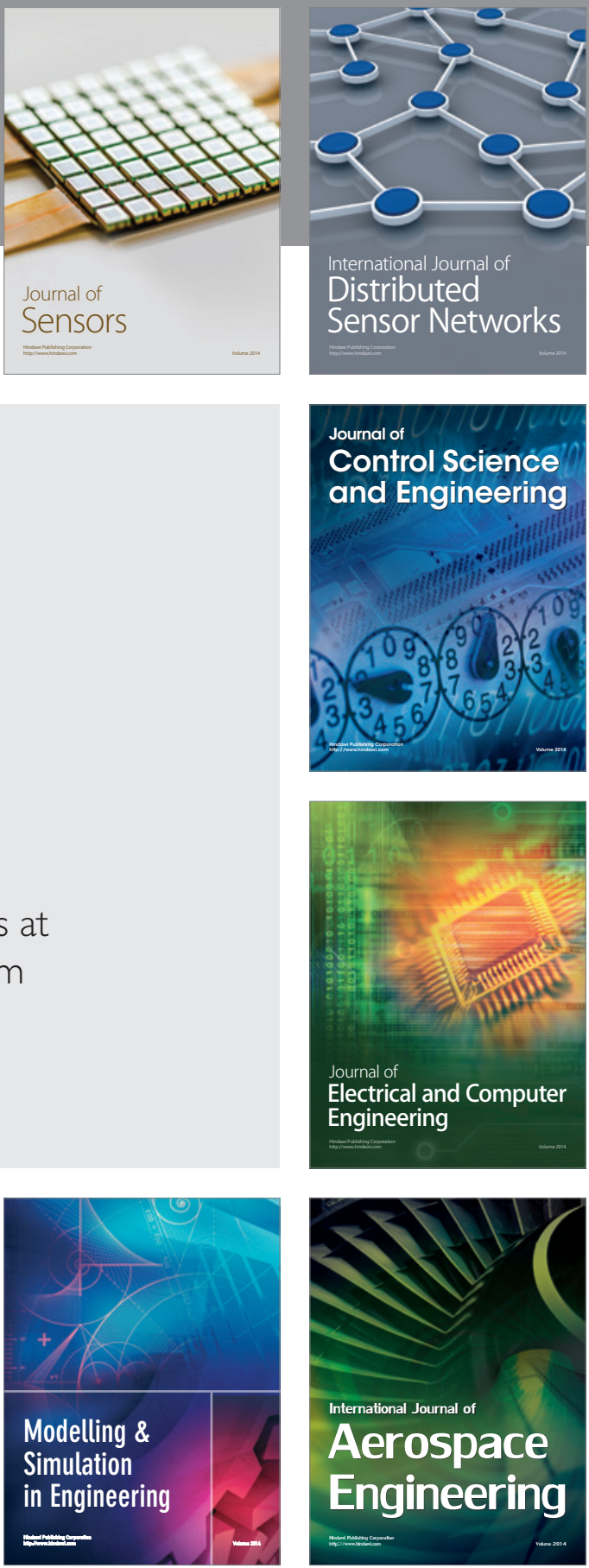

Journal of

Control Science

and Engineering
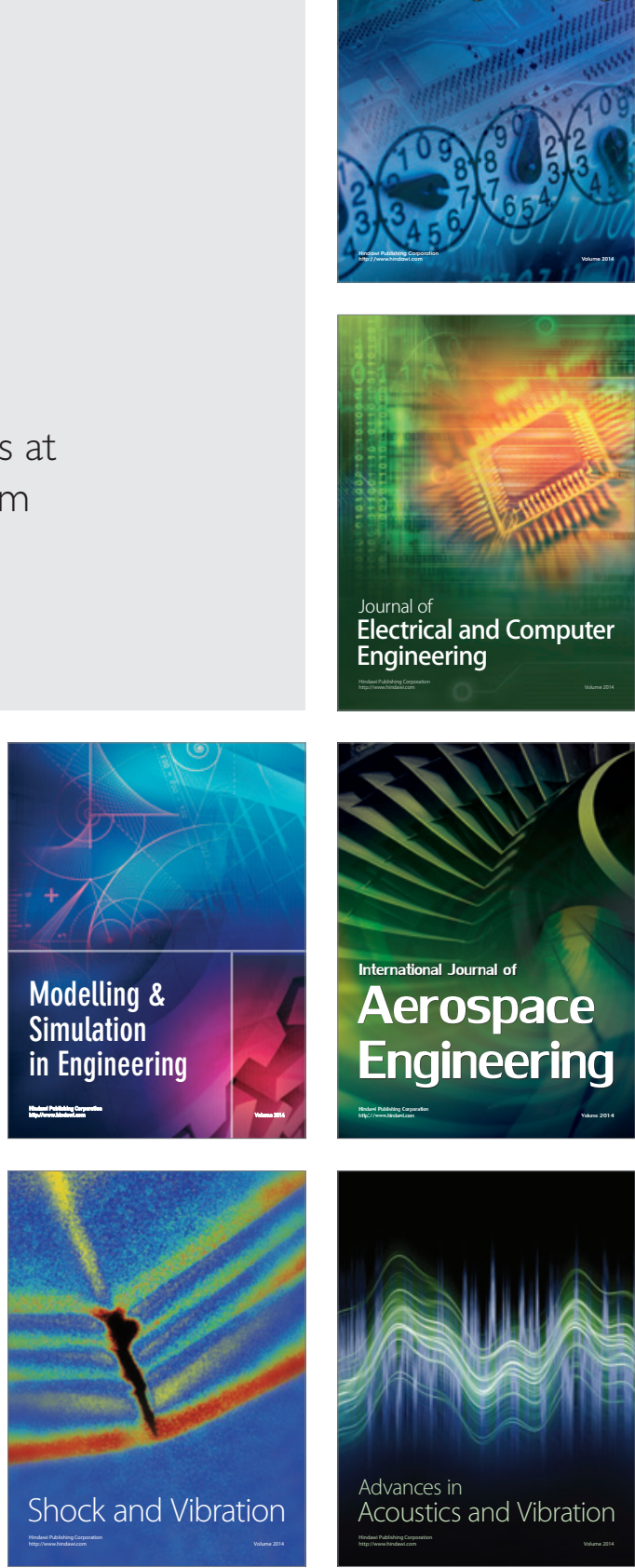